\title{
Case Study Research Methodology
}

\section{(C) 2011 Mark Widdowson}

\begin{abstract}
Commenting on the lack of case studies published in modern psychotherapy publications, the author reviews the strengths of case study methodology and responds to common criticisms, before providing a summary of types of case studies including clinical, experimental and naturalistic. Suggestions are included for developing systematic case studies and brief descriptions are given of a range of research resources relating to outcome and process measures. Examples of a pragmatic case study design and a hermeneutic single-case efficacy design are given and the paper concludes with some ethical considerations and an exhortation to the TA community to engage more widely in case study research.
\end{abstract}

\section{Key words \\ Case study design, case study research}

\section{Introduction}

Case study methodology is becoming increasingly influential in psychotherapy research. Although therapists tend to write case studies as part of their training, there is a definite need for the training of psychotherapists in case study research methodology and developing the skills needed to design rigorous and scientific systematic case studies. The aim of this article is to provide the reader new to case study research with a background in the method to assist them in creating and developing case study research and of contributing this to the TA research literature. Although written for a psychotherapy audience, the key principles of the methodology can be extracted by practitioners from other fields and applied to their own situation.

The development of psychotherapy has been influenced from the beginning by the writing and publishing of case studies. Freud's $(1901,1909)$ now famous cases were highly significant in the development of psychoanalysis. Case Studies were also influential in the development of behavioural therapy (Wolpe, 1958), and indeed most modalities of psychotherapy are often influenced by several key case studies which triggered innovative thinking or methods in the originator(s) of the therapy, or cases that were used to test out and verify the effectiveness of the new therapy, or to explain key features of the therapy and how it works to a wider audience (see also Berne, 1961).

"In the practice of psychotherapy, the most basic unit of study is the 'case'"(Eels, 2007). Single-case studies that allow for the examination of the detailed unfolding of events across time in the context of the case as a whole represent one of the most pragmatic and practiceoriented forms of psychotherapy research. (Fishman, 1999, 2005)" (Iwakabe and Gazzola, 2009. p.601). Within psychotherapy, a case study may be of a single episode within a session, a single session, a particular phase or 'chunk' of therapy or an overview of the entire therapy.

Despite the historical significance of case studies in the development of psychotherapy, there are few case studies published in modern psychotherapy books and journals. Within the TA literature there is a lack of detailed case studies which provide the reader with a clear sense of the work, and sufficient information to come to their own conclusions regarding the outcome. Of the case studies which are available, like the case studies of Freud, they each tell a story, but do not provide the required evidence needed for scientific inquiry or for reliable conclusions to be drawn from the presented cases.

The psychotherapy research community has recently begun to turn its attention to case study methodology and how this research approach can be rigorously enhanced so that reliable conclusions can be identified from the studies. Case study methodology is growing in significance as a method highly suitable for use in psychotherapy research and the view is gaining momentum that case study methodology will develop into the next important area of psychotherapy research (McLeod, 2010). Kiesler (1983) states "Studies 
seriously pursuing these [psychotherapy] changeprocess goals cannot attain them by use of traditional, rigorous experimental or nomothetic designs. Instead, what seems to be most appropriate and necessary are small $N$ or single-case studies." (Kiesler, 1983. p.13). Certainly well-constructed and thorough case studies can be used as reliable evidence for the impact of the therapy in effecting change.

\section{The strengths of case study methodology}

"Single-case research is best viewed as a sub-class of intrasubject research in which aggregation across cases is avoided and the generality of one's findings is addressed through replication on a case-by-case basis." (Hilliard, 1993: 373-4)

The case study offers a rich method for investigating and researching a single case. The effectiveness of the approach being researched can be verified by replication of outcomes across similar cases. Due of the level of detail kept in the case record, outcomes of different but similar cases can be compared, and the specific variables which might have impacted upon the difference in outcome can then be investigated separately. In psychotherapy research, case study methodology has the advantage of being closely linked to therapy as it is usually delivered. The case study is measuring what actually happens in the therapy situation, rather than creating some tightly controlled situation that may bear little resemblance to 'therapy as usual'.

Case studies have the advantage of providing the reader with a clear sense of the person of the client, the therapist, the therapy and of the outcome(s). One criticism of methods of psychotherapy research, such as randomised controlled trials (RCTs), is that they focus on large, generalised quantitative data, and that essentially the findings are reduced to a table of numbers without accounting for the complexity of the therapy and without examining the different factors that have impacted on the case. Elliott (2001) describes how such methods of research (such as RCTs) are 'causally empty', in that they do not provide sufficient data for clear causal explanations to be drawn as to how or why a particular therapy has generated a particular change. In contrast, detailed case studies which account for and include a range of data (including factors from within and outside the therapy e.g. changes in a client's circumstances) enable the researcher/reader to draw more convincing causal explanations from the case.

"For researchers, the closeness of the case study to real-life situations and its multiple wealth of details are important in two respects. First, it is important for the development of a nuanced view of reality, including the view found at the lowest levels of the learning process and in much theory, that human behaviour cannot be meaningfully understood as simply rule-governed acts.
Second, cases are important for researchers' own learning processes in developing the skills needed to do good research. If researchers wish to develop their own skills to a high level, then concrete, context-dependent experience is just as central for them as to professional learning of any other specific skills" (Flyvbjerg, 2006. p.223)

Case study methodology is also highly relevant to a postmodern perspective to psychotherapy with its accounting for a range of factors in the work. "Predictive theories and universals cannot be found in the study of human affairs. Concrete, context-dependent knowledge is, therefore, more valuable than the vain search for predictive theories and universals" (Flyvbjerg, 2006: 224). Case studies generate context-dependent knowledge which is an appropriate form of knowledge base in social sciences and disciplines based on observation and understanding of human behaviour and interaction in context. Flyvbjerg emphasises "in the social and human behavioural sciences...contextdependent knowledge and experience... (is at) the very heart of expert activity" (Flyvbjerg, 2006. p222).

This position is based on the ability of experts to move beyond rigid, rule-bound approaches to ones which embrace complexity and require the higher level of theoretical and intellectual abstraction which is relevant in professional training programmes. Flyvbjerg goes on to state that presenting and discussing case studies is an important teaching method for imparting practical skills and promoting the development of professional decision making skills. Certainly, accounting for complexity in an individual's life and the interaction of various factors which may have influenced the change process, learning and refining the processes of theoretical and intellectual abstraction and assessing the often subtle impact of interventions, are key aspects of psychotherapy training.

\section{Common Criticisms of Case Study}

\section{Methodology}

It is often believed that because the cases are so specific, one cannot make meaningful generalisations from case studies and that other methods are more suited to hypothesis testing and theory building. However this view is not accurate as case studies provide a wonderful opportunity for the researcher to develop explanatory hypotheses or test existing or new theory (McLeod, 2010).

Certainly it is true that it is not possible to generate inferential statistics from a single case or indeed from a small number of cases; however it is possible to use simple descriptive statistics to enable the reader to draw logical conclusions regarding the outcome(s) of the therapy, and replication of the case methodology can result in large databases being constructed which would enable inferential statistics to be generated. If, for 
example, data from a series of 30 similar or matched cases were placed on a database, it would be possible for a researcher to generate inferential statistics which would support the process of generalisation from the cases.

Flyvbjerg (op cit) summarises the main criticisms of case study methodology as being based on issues relating to theory (and the perception that case studies are inappropriate for theory-building), reliability and validity.

\section{Case Studies for Theory Building}

One criticism of case studies is that as they are so specific, it is impossible to generate theory from one single case; however it could be argued that case studies have been a primary source of theory building within psychotherapy since its origin. A particular theorist may have had a theory hunch, and then on the basis of their experiences with a particular case, tested their theory. The process of repeated theory testing then strengthened the theoretical proposition. Examples where cases did not conform to the theory, or contained unexplained features required the theorist to develop a theory to explain the unaccounted phenomena. The case(s) can then be analysed to discover if theory can be meaningfully applied to the case(s) in question, or if indeed new theory is needed to explain the phenomena which are being described. (Flyvbjerg, 2006). Stiles (2007), has specifically discussed the strength of using case studies for theory-building. For Stiles, observation is a key feature of theory-building - ". . . in any scientific research, observations change theories. They may confirm or disconfirm or strengthen or weaken the theory. More constructively, the changes may involve extending, refining, modifying or qualifying the theory... observations permeate the theory... Thus a theory is not a fixed formula, but a growing and changing way of understanding." (Stiles, 2007: p.123)

Case studies can be used to identify processes which may not have hitherto been recognized within therapy, or within that therapeutic modality or specific ways in which the particular problem was addressed by the therapist and client which confirm, develop, disconfirm or extend the existing theoretical positions of that particular theoretical approach. They can be used to strengthen or weaken certain theoretical propositions or test the validity of theoretical constructs and to develop or challenge existing theory (McLeod, 2010).

"The case study is useful for both generating and testing of hypotheses but is not limited to these research activities alone" (Flyvbjerg, 2006. p.229). "The case study is ideal for generalizing using the type of test that Karl Popper (1959) called 'falsification', which in social science forms part of critical reflexivity. Falsification is one of the most rigorous tests to which a scientific proposition can be subjected. If just one observation does not fit with the proposition, it is considered not valid generally and must therefore be either revised or rejected" (Flyvbjerg, 2006. p.227-8). We can see from this that case studies can have a valuable role in falsification of theory - a single case which does not 'fit' the proposed theory would require that theory to be revised (perhaps including specific indicators about what sort of factors might mean the theory was accurate or the factors which might mean the theory would not apply) or may result in the theory being rejected as inaccurate or irrelevant.

\section{Internal versus External Validity}

High internal validity requires the controlling of as many variables as possible to create as 'pure' a scientific experiment as possible. In large-scale studies into psychotherapy (such as RCTs of manualised therapies) variables are tightly controlled, and the client group being studied is clearly defined using a set of inclusion and exclusion criteria. In such studies, the level of internal validity is considered to be high, due to the rigour of the experimental design. However, the results of many such studies cannot be easily generalised to 'regular clinical practice' and so they are considered to have low external validity, which relates to the generalisability of the findings. An example of this might be a study which investigates the outcome of 'manualised treatment $X$ ' with a tightly-defined client population. The study might suggest that the therapy was effective but it can only be concluded that the therapy was effective when delivered in that manner and with that specific client group.

Case study methodology would generally be low in internal validity (due to the absence of experimental controls), but high in external validity and would provide findings useful to a large number of therapists who would be able to extrapolate from the findings of the case study into their own practice. In this sense, case study methodology generates research which is 'user friendly' and closes the research-practice gap. This refers to the perceptions of many therapists that research findings are inaccessible and irrelevant to their practice or their usual client demographics. Cases do not necessarily need to report positive results for the case study to be useful to therapists in practice or for the purposes of theory building.

Strupp (1980a,b,c,d) conducted a cross-case analysis study of both good outcome and poor outcome cases to examine which factors could be identified as having an influence in whether a case would have a good or poor outcome. Strupp concluded that agreement between the client's views about the nature and origin of their problems and what would be helpful to them and the therapist's way of working with the client was associated with better outcomes. Furthermore, in the poorer-outcome cases, the therapist often did not know how to respond effectively to the client's frustration or hostility arising from the mis-match between client and therapist expectations or attributions of the origin of the 
problem and the most helpful approach to resolution of these problems.

\section{Issues in Case Selection}

Flyvbjerg (2006) identifies two primary strategies in case selection; random selection and informationoriented selection. The intention with random sampling is that the sample is sufficiently representative of the population being studied to enable generalisation of findings to take place. With information-oriented selection, cases are carefully chosen for their significance, in that they may be extreme cases which may reveal or suggest certain findings, or critical cases which can be exemplars, or 'typical' cases from which generalisations can be drawn through logical deduction.

Other issues in case selection can include selecting cases which are theoretically interesting, or ones which either confirm or challenge theory, or innovative cases which might utilise new and original techniques or approaches. A series of cases can be used for comparison, using cases which have either similar or diverse characteristics or good versus poor outcomes so the researcher can seek to identify the crucial variables which influenced the outcome.

Issues of Objectivity in Reporting

Clinical case studies are written by the therapist and as such are inevitably influenced by the therapist's subjectivity and (unconscious) bias. What is usually missing in a clinical case study is the client's perspective and/or data which can be used for the purposes of triangulation (for example data from standardised outcome measures can be used to support a claim in a case study that the client experienced positive change).

Flyvbjerg (2006) discusses the issue of 'bias toward verification', or researcher allegiance in case study methodology. As therapists, we (understandably) want to see or portray a positive outcome in the cases we present. Even the best-intentioned of therapists can unconsciously be selective in the information they present to show their work in a positive light. Also, one can reasonably expect that a therapist of any particular theoretical orientation would want to paint their particular type of therapy in a positive light and therefore introduce issues relating to such researcher allegiance.

The issue of lack of objectivity on the part of the researcher is considered to be a common critique of case study methodology. However Flyvbjerg (2006) argues that case study methodology most commonly results in a challenging of the researcher's "preconceived views, assumptions, concepts, and hypotheses" (p.235). Flyvbjerg considers that such challenging of underlying assumptions comes about due to the rich data revealed in case study methodology that challenges the researcher's theories by finding exceptions to the rule.

Some methods of case study methodology, such as Elliott's (2001; 2002) Hermeneutic Single-Case Efficacy Design (HSCED - see below for more details) address the issue of objectivity by recruiting an adjudication panel to critique the findings of the case study.

\section{Research questions which can be}

\section{investigated by case studies}

The process of research begins with the researcher deciding what questions they want to investigate in their study, or by the researcher identifying questions that the case under investigation raises. John McLeod offers a list of a number of questions which the therapist can use to help them guide and design their case study research

"Outcome questions: How effective has therapy been in this case? To what extent can changes that have been observed in the client be attributed to therapy?

Theory-building questions: How can the process of therapy in this case be understood in theoretical terms? How can the data in this case be used to test and refine an existing theoretical model?

Pragmatic Questions: What strategies and methods did the therapist use in this case that contributed to the eventual outcome? How were the therapeutic methods adapted and modified to address the needs of this specific client? What are the principles of good practice that can be derived from this case?

Experiential or narrative questions: What was it like to be the client or therapist in this case? What is the story of what happened, from the client's or therapist's point of view?" (Mcleod, 2010 p.21 reproduced with permission)

\section{Types of Case Studies}

\section{Clinical Case Studies}

(Iwakabe and Gazzola, 2009)

A clinical case study is a narrative account of the therapy, written by the therapist. It is through clinical case studies that psychotherapy as a profession was developed, as discussed above. Clinical case studies are engaging to the reader, and particularly useful in the teaching of psychotherapy, where trainee therapists can learn about how to implement therapy with real-life clients, and how to navigate some of the problems which inevitably occur through the therapy process. The writing of clinical case studies is also a key feature of most psychotherapy training courses and some accreditation processes. Whilst clinical case studies are important and valuable for the development of psychotherapy, because they do not rely on 
methodological guidelines for research, and are based on the therapist's (subjective) account of the therapy, clinical case studies are not reliable for research purposes (Iwakabe and Gazzola, 2009).

\section{Experimental Case Studies}

(Iwakabe and Gazzola, 2009)

Often known as $N=1$ studies, experimental case studies are methods for "testing hypotheses about treatment effects and may be considered alternatives to largescale outcome research (e.g. Barlow \& Hersen, 1984). The aim of experimental case studies is to record and address specific changes observed in clients that are attributable to the administration of specific interventions. A standard test or behaviour assessment is conducted regularly, and changes are compared with a baseline of target behaviours and other physiological indexes obtained before introducing the treatment" (Iwakabe and Gazzola, 2009 p.602) Given the emphasis on behavioural change, and the outcome of specific, targeted interventions, the $\mathrm{N}=1$ model has been almost exclusively used for the research of behavioural therapies.

McLeod (2001) describes some strengths of this approach as being the use of:

1. time-series analysis as a means of assessing change;

2. of baseline measures to establish the stability of a problem before therapy begins (which may include the use of a number of reliable tools to measure outcome variables); and

3. a methodology that can be readily integrated into routine practice (including a detailed description of the intervention).

The $\mathrm{N}=1$ format has been extensively used by behavioural therapists, who would measure a specific behaviour and then apply a particular intervention and then measure the behaviour after the intervention application. For example, a client may record the frequency and severity of their self-harming prior to therapy to obtain baseline information regarding the extent of the client's problem and to establish the stability of the problem. The therapist would then use a manualised intervention with the client, and frequency and severity of self-harming following the intervention would be measured. This might take place over a series of sessions, which would often be spaced to enable the researcher to see clearly when a significant intervention had been introduced into the therapy process. "The purpose of valid and reliable measurement in $\mathrm{N}=1$ studies is to make it possible to make statements about what changed in response to a specific intervention at a specific time" (McLeod 2010: 119).

Whilst the $\mathrm{N}=1$ design is a useful approach for measuring the impact of specific therapeutic interventions or the effectiveness of certain techniques, it does not account for 'soft' factors in the therapy, such as the impact of the therapeutic relationship on the change process, or the impact of external factors and extra-therapy events in facilitating change.

Naturalistic/ Systematic Case Studies

(Iwakabe and Gazzola, 2009)

Systematic case studies rectify many of the methodological problems associated with clinical case studies. The means by which these methodological problems are resolved is through the use of "data . . . gathered from multiple sources, such as questionnaires, therapist and observer ratings, and participant interviews, to construct a rich and comprehensive account or case summary, which is then triangulated in order to examine whether different sources of data converge." (Iwakabe and Gazzola, 2009 p.602-3). Frequently, a team of researchers is used for the purposes of data analysis, which may include a panel, or 'jury' of researchers exploring different interpretations of the findings (Elliott, 2002; Iwakabe and Gazzola, 2009; McLeod, 2010). Systematic case studies represent perhaps the most appropriate and accessible method for developing the research evidence-base for TA.

\section{Developing a systematic case study}

Well-designed systematic case studies enable the researcher to account for additional factors and complexity in the client's life, such as the influence of external or extra-therapy changes (e.g. changes in relationships or work-related changes) and other variables in a way that is not present in large-scale quantitative research. Case studies which include complex data and a 'rich' description of the client account for the context and uniqueness of the individual in a way that is philosophically consistent with TA and other humanistic approaches to psychotherapy.

A case study would generally have a fairly detailed narrative throughout. In order to capture some of the richness and 'flavour' of a case, the narrative is essential to explore the context-dependent factors within the case. Within psychotherapy research the narrative would generally include details of the client's history and presenting problem, together with a detailed account of the therapy work, to enable the reader to determine whether the interventions did indeed result in the described effect, or if alternative explanations can be applied. (McLeod, 2010)

Kazdin (1981) identified a number of characteristics of case studies suitable for research purposes which allow the reader to draw reasonable conclusions from the evidence presented. These characteristics are:

1. Use of reliable and valid methods of measuring the client's change;

2. Regular, repeated measurement of specific relevant outcome variables (for example measurement of symptoms using a standardised outcome measure taken at weekly or monthly intervals); 
3. Assessment or measurement of stability of the client's problem prior to commencing therapy;

4. Marked effect on the identified problem/ symptoms following the commencement of therapy;

5. Replication of the results with multiple cases.

One can see that if a client's problems have been stable before therapy and that the measurement of these variables (such as symptoms) using reliable methods at regular intervals throughout the therapy demonstrates that positive change has indeed occurred, then one can speculate that the therapy has probably contributed to the client's improvement. If this is repeated with a number of similar clients, one can reasonably conclude that such therapy is an effective intervention for the treatment of that specific problem.

The use of reliable and validated outcome measures is a key feature of Kazdin's argument, as these tools go beyond anecdotal reports to add a degree of scientific validation and therefore lend credibility to the argument that positive therapeutic change has indeed taken place. Identification of the stability of the problem prior to commencing therapy also addresses concerns that the client's problems were subject to fluctuation and that any change may have taken place as a result of spontaneous remission.

Replication of findings also addresses the issue of the change occurring by chance, and enables findings from the case series to be generalised. For example, if through repeated systematic case study research one can see that a particular therapy shows repeated effectiveness in the treatment of a specific problem with a wide range of clients with an identical problem, one can reasonably assume that the therapy being researched has some validity for the treatment of that specific problem. (McLeod, 2010)

\section{Collecting 'The Rich Case Record'}

A thorough, systematic case study will collect a range of data to enable the case to be analysed from a range of perspectives. This data might include

- Outcome measures (such as CORE, PHQ-9, Beck Depression Inventory etc. See below for more information)

- Process data

- Session recordings and transcripts

- Interviews (of either or both client and therapist)

- Therapist notes

- Client notes/ diaries

- $\quad$ other information (for example, referral letters)

\section{Resources for prospective researchers}

With research, there is generally no need to 're-invent the wheel', and practitioner-researchers who wish to conduct systematic case studies have a number of tools freely available which they can use to monitor both the process and the outcome of the therapy. One advantage of using such existing tools is that they have already been validated and have been used in previous research.

\section{Outcome Measures}

\section{CORE (Clinical Outcomes for Routine Evaluation)}

(Barkham, et al., 2006)

The CORE system is in wide use within the UK as an evaluation system for examining outcomes of therapy. The standard tool is the CORE-OM, a 34-item selfreport measure which has four sub-scales; Well-Being, Functioning, Problems and Risk. There are two shorter measures, CORE-10 and CORE-18 which can also be used to measure global distress and change. It is free to use and is available from www.coreims.co.uk

\section{PSYCHLOPS (Psychological Outcome Profiles)}

(Ashworth et. al, 2004)

PSYCHLOPS is a short client-generated outcome measure consisting of three domains; the problem domain (clients are asked to describe and rate their main problems), the functioning domain (clients describe and rate what they have problems doing as a result of their presenting problems), and the well-being domain (clients give a subjective rating of their overall well-being). It is available from www.psychlops.org.uk

PQ (Personal Questionnaire) (Eliott et al. 1999)

The simplified personal questionnaire $(P Q)$ is a clientgenerated measure based on the particular presenting problems the client wishes to address in therapy. The $P Q$ can be used every week to measure progress and change with the client's problems. It is free to use and is available from Error! Hyperlink reference not valid.

PHQ-9 (Patient Health Questionnaire) (Kroenke et. al 2001)

PHQ-9 is a nine-item self-report measure which is based on the diagnostic criteria for major depressive disorder from the American Psychiatric Association's Diagnostic and Statistical Manual (APA, 1994: DSM-IV). It is free to use, and is available from www.phqscreeners.com or from Error! Hyperlink reference not valid.

GAD-7 (Generalised Anxiety Disorder) (Spitzer, et al. 2006)

A seven-item self-report measure based on the DSM-IV (APA, 1994) diagnostic criteria for generalised anxiety disorder. It is free to use and available from

www.phqscreeners.com 
The change interview is a structured qualitative research tool, which explores the client's own views on their change process and the changes they have experienced as a result of being in therapy. The change interview can be administered at the end of the therapy or at intervals during the course of the therapy. It is recommended that this interview is used in conjunction with the Personal Questionnaire (PQ). It is free to use and is available from

http://www.experiential-researchers.org/

instruments.html

\section{Process measures}

Session Evaluation Questionnaire (SEQ(Stiles, 1980; Stiles, et al. 2002)

The SEQ is a 21 -item self-report scale in which the client evaluates the session according to dimensions of depth, smoothness, positivity and arousal. It is free to use and is available from

http://www.users.muohio.edu/stileswb/

Working Alliance Inventory (WAI short form) (Hatcher and Gillaspy, 2006)

The WAl is a 12-item self-report form and has specific forms for the therapist and client. As a measure, it is based on Bordin's (1979) conceptualisation of the working alliance as being comprised of tasks, bonds and goals. It can be used to measure the strength of the working alliance - a factor which is widely accepted as critical to the outcome of the therapy. You will need to get permission to use this tool in research. The form and contact details for obtaining permission to use the WAI can be obtained from

http://www.educ.sfu.ca/alliance/allianceA/waidoc/Short $\underline{\text { WAll }}$

Helping Alliance Questionnaire (HAq-II) (Luborsky, et al. 1996)

A 19 item questionnaire, similar to the Working Alliance Inventory. It is free to use and is available from http://www.med.upenn.edu/cpr/instruments.html

Helpful Aspects of Therapy (HAT) (Llewelyn, 1988)

The HAT is a short open-ended questionnaire which the client completes after the session to evaluate both helpful and hindering aspects of the therapy session and to identify particular events within the session or interventions which were most helpful. It is free to use and is available from

http://www.experiential-researchers.org/ instruments.html design

Pragmatic case studies

Fishman (2005) has described guidelines for the publication of case studies which involves a rich description of the client and their presenting problems which incorporates the therapist's theoretical approach and preferred intervention methods, the practitioner's professional experience and competence in dealing with similar cases, and which discusses and accounts for previously published research that is relevant to the case being investigated (Fishman, 2005; Iwakabe and Gazzolla, 2009.)

The title 'pragmatic' was chosen by Fishman (1999) as a concept based on the philosophical tradition of pragmatism, which holds "that it is not satisfactory to regard knowledge as consisting of a set of abstract ideas. Instead knowledge is more appropriately understood as a capacity to take effective action within a specific context" (McLeod 2010: 94).

Fishman was interested in developing a case study method which emphasises what the practitioner actually did, but locates this within a context and requires researcher reflexivity. Developing the work of Peterson (1991), Fishman (2005) designed a format for case studies submitted to the online journal Pragmatic Case Studies in Psychotherapy (PCSR) which uses this standard reporting format for all published case studies, and provides a means for the published case studies to be peer-reviewed. The format used in PCSR is as follows:

1. Case context and method

2. The client

3. Guiding conception with research and clinical experience support

4. Assessment of the client's problems, goals, strengths, and history

5. Formulation and treatment plan

6. Course of Therapy

7. Therapy monitoring and use of feedback information

8. Concluding evaluation of the process and outcome of therapy

\section{References}

This design of case study reporting provides the reader with a comprehensive account of the therapy and information regarding the therapist's experience and theoretical perspective, together with their formulation, treatment plan and information regarding the process and outcome of the therapy. The intention is to provide a rich account of the therapy which can be analysed and compared with other similar cases, and which provides sufficient detail so that 'active ingredients' and 
technical aspects of the therapy can be readily identified or the findings could be replicated with a similar client/ therapist context.

McLeod's (2010) critique of the pragmatic approach to case study reporting is that it focuses on the technical aspects of the therapy, perhaps at the expense of factors such as the therapeutic relationship, the therapist's own subjectivity, and client feedback. McLeod goes on to suggest that this approach would be strengthened by the inclusion of more contextual information, for example regarding the supervision the therapist received on the case, what limitations surrounded the work, what extra-therapy factors might have impacted on the outcome and so on.

Hermeneutic Single-Case Efficacy Design (HSCED)

Elliott's Hermeneutic Single-Case Efficacy Design (HSCED) (Elliott, 2001; 2002) is a case study design method which employs a number of strategies with established criteria guidelines to enable the researcher(s) to make interpretations about the efficacy of the therapy in relation to the case in question.

HSCED employs an adjudication process whereby two contrasting interpretations of the case are built in a manner similar to debating societies and perhaps using a team of researchers - one team would build an argument that the therapy was effective (the affirmative case) and the second team would build an argument that the therapy was not effective or that the client's changes did not come about as a result of the therapy (the sceptic case). The arguments of each stance are put forward and challenged by the opposing side. Rebuttals are then formed to these challenges. The arguments and rebuttals presented by both the affirmative and sceptic teams can then be put to an adjudication panel who, similar to a judge and jury in court, would reach a verdict regarding the outcome of the case. The adjudicating panel would adopt the same criteria as is used in civil law - namely that something has only to be established as likely when considered by the 'balance of probabilities' as opposed to the 'beyond reasonable doubt' (almost definite) criterion of criminal law.

HSCED also accounts for non-therapy explanations for any change that might have taken place. For instance, the beneficial impact that a new job might have on someone's self-esteem and stress levels might be considered as opposed to making statements that such changes in the client came about solely as a result of psychotherapy. These non-therapy explanations are brought into the sceptic argument, as are a number of other factors which may be used to draw the conclusion that the client's changes did not occur as a result of therapy. This process of examining the different factors involved in the case, exploring alternative hypotheses, and including the affirmative and sceptic cases to the published case study, adds credibility to any claims that the client changed as a result of the therapy. The credibility of the claims is also enhanced where the adjudication panel are independent researchers who have no theoretical allegiance to the method investigated (for example, inviting a number of non-TA therapists to participate in the adjudication process and including their 'verdict' in the published article).

\section{Ethical Issues in Case Studies}

In all research, research participants need to be free to make the decision as to whether to participate or not in the research from a position of informed consent. This is a difficult question, as it can be argued that the client cannot truly know precisely what they are entering into at the outset. One way this can be addressed is to consider consent as an on-going process rather than a one-off event.

A common anxiety amongst prospective researchers is the worry about how the writing of the case study or the research process will intrude upon the therapy process. It is inevitable that the research process will influence the case to some extent. Perhaps in this instance, it might be better (to paraphrase Berne) to ask 'how has the research process influenced this case?' rather than 'did the research process influence this case?' Despite the fact the research will impact on the work, it is possible that this effect can be beneficial to both the client and therapist.

Confidentiality is an issue in case studies, as large amounts of detail about the client's life may be published, thus compromising the client's anonymity. Sufficiently disguising the case without obscuring significant or important factors and details can be difficult. Ethical case study research involves inviting the client to read and comment on the finished case study, giving the client the opportunity to request that certain information be removed, and obtaining the client's consent for the case to be published.

\section{Conclusions and recommendations to the}

\section{TA community}

Case study methodology is a small-scale research method which can readily be used by practitionerresearchers from the TA community to test and develop TA theory and to explore the processes and outcomes of TA psychotherapy in practice.

Trainees can be easily and routinely taught the principles of critical inquiry as used in case study methodology to evaluate and refine their work by inviting them to reflect on questions such as 'How is this a good or poor outcome case? What criteria can be used to define this? What are the strengths and limitations of this case? If the outcome was poor, what factors contributed to this? What could have been done 
differently?' The development of such critical inquiry and evaluation skills will have a direct effect on increasing the capacity of trainees to accurately evaluate their work in day to day practice.

Furthermore, the requirement to use and include critique and data from process or outcome measures and/or 'the client's voice' (by including feedback from the client relating to the change process, their experiences of therapy and their perspectives on the outcome of the therapy) in all case studies both in psychotherapy training programmes and within the CTA examination process will rapidly and substantially increase the development of research expertise within the TA community. This will inevitably result in an increase in the amount of published case study research which contributes to the evidence base for TA.

Mark Widdowson, Teaching and Supervising Transactional Analyst (Psychotherapy), Associate Director, The Berne Institute, PhD student, University of Leicester, can be contacted on: mark.widdowson1@btopenworld.com

\section{References}

American Psychiatric Association (Ed.) (1994) Diagnostic and Statistical Manual of Mental Disorders- fourth edition. Washington, DC: American Psychiatric Association

Ashworth M, Shepherd M, Christey J, Matthews V, Wright K, Parmentier H, Robinson S, Godfrey E. (2004) 'A client-centred psychometric instrument: the development of 'PSYCHLOPS' ('Psychological Outcome Profiles')'. Counselling and Psychotherapy Research,;4:27-33.

Barkham, M., Mellor-Clark, J., Connell, J., and Cahill, J. (2006) 'A core approach to practice-based evidence: a brief history of the origins and applications of the CORE-OM and CORE system'. Counselling and Psychotherapy Research, 6(1): 3-15.

Berne, E. (1961) Transactional Analysis in Psychotherapy. New York: Souvenir Press.

Bordin, E.S. (1979) 'The generalizability of the psychoanalytic concept of the working alliance' Psychotherapy, Theory Research and Practice, 16: 252-60.

Eels, T.D. (2007) 'Generating and generalizing knowledge about psychotherapy from pragmatic case studies'. Pragmatic Case Studies in Psychotherapy, 3(1): 35-54.

http://pcsp.libraries.rutgers.edu

Elliott, R., Fischer, C.T., and Rennie, D.L. (1999) ‘Evolving guidelines for the publication of qualitative research studies in psychology and related fields'. British Journal of Clinical Psychology, 38: 215-9.
Elliott, R. (2001) 'Hermeneutic Single Case Efficacy design: An Overview' in K.J. Schneider, J.Bugental and J.F. Pierson (eds) The Handbook of Humanistic Psychology: Learning Edges in Theory, Research and Practice. Thousand Oaks, CA: Sage.

Elliott, R. (2002) 'Hermeneutic Single Case Efficacy Design'. Psychotherapy Research, 12: 1-20.

Fishman, D.B. (1999) The Case for a Pragmatic Psychology. New York, New York University Press.

Fishman, D.B. (2005) ‘Editor's introduction to PCSP- from single case to database: a new method for enhancing psychotherapy practice'. Pragmatic Case Studies in Psychotherapy, 1(1): 1-50. http://pcsp.libraries.rutgers.edu

Flyvbjerg, B. (2006) 'Five misunderstandings about case-study research'. Qualitative Inquiry, 12(2):219-45.

Freud, S.(1901/ 1979) 'The case of Dora' Pelican Freud Library, Vol. 8: Case Histories.l. Harmondsworth: Penguin.

Freud, S. (1909)/1979) 'Notes upon a case of obsessional neurosis (the 'Rat Man')'. Pelican Freud Library, Vol. 9: Case Histories II. Hardmonsworth, Penguin. Hilliard, R.B. (1993) 'Single-case methodology in psychotherapy process and outcome research'. Journal of Consulting and Clinical Psychology, 61(3): 373-80.

Hatcher, R.L. and Gillaspy, J.A. (2006) 'Development and validation of a revised short form of the Working Alliance Inventory'. Psychotherapy Research, 16: 12-25.

Iwakabe, S. and Gazzola, N. (2009) 'From single-case studies to practice-bases knowledge: aggregating and synthesizing case studies'. Psychotherapy Research, 19: 601-11.

Kazdin, A.E. (1981) 'Drawing valid inferences from case studies'. Journal of Consulting and Clinical Psychology, 49: 183-92.

Kiesler, D. J. (1983, October). The paradigm shift in psychotherapy process research. Summary discussant paper presented at the National Institute of Mental Health Workshop on Psychotherapy Process Research, Bethesda, MD.

Kroenke, K., Spitzer, R.L. and Williams, J.B. (2001) 'The PHQ9: validity of a brief depression severity measure'. Journal of General Internal Medicine;16(9):606-13.

Llewelyn, S. (1988)'Psychological therapy as viewed by clients and therapists'. British Journal of Clinical Psychology, 27: 22338.

Luborsky, L., Barber, J.P., Siqueland, L., Johnson, S., Navajits, L.M., Frank, A. and Daley, D. (1996) 'The Revised Helping Alliance Questionnaire (HAq-II): psychometric properties'. Journal of Psychotherapy Practice, 5: 260-71. 
McLeod, J. (2010) Case Study Research in Counselling and Psychotherapy. London, Sage.

Peterson, D.R. (1991) 'Connection and disconnection of research and practice in the education of professional psychologists', American Psychologist, 46: 422-9.

Spitzer, R.L., Kroenke, K.and Williams, J.B. (2006) 'A brief measure for assessing generalized anxiety disorder: the GAD-7'. Archives of Internal Medicine, 166(10): 1092-7.
Stiles, W. B.(1980) 'Measurement of the impact of psychotherapy sessions'. Joumal of Consulting and Clinical Psychology, 48: 176-85.

Stiles, W.B. (2007) 'Theory-building case studies of counselling and psychotherapy'. Counselling and Psychotherapy

Research, 7:122-7.

Stiles, W.B., Gordon, L.E., and Lani, J.A.(2002) 'Session evaluation and the Session Eels, T.D. (2007) 'Generating and generalizing knowledge about psychotherapy from pragmatic case studies'. Pragmatic Case Studies in Psychotherapy, 3(1): 35-54. http://pcsp.libraries.rutgers.edu 